\title{
Evolution of nonlinearly coupled drift wave-zonal flow system in a nonuniform magnetoplasma
}

\author{
D. J OVANOVIC ${ }^{1}$, P. K. SHUKLA ${ }^{2}$ and B. ELIA S S O N ${ }^{3}$ \\ ${ }^{1}$ Institute of Physics, 11001 Belgrade, Serbia \\ (djovanov@ipb.ac.rs) \\ ${ }^{2}$ Institut für Theoretische Physik IV, Fakultät für Physik und Astronomie, \\ Ruhr-Universität Bochum, D-44780 Bochum, Germany \\ ${ }^{3}$ Institut für Theoretische Physik IV, Fakultät für Physik und Astronomie, \\ Ruhr-Universität Bochum, D-44780 Bochum, Germany
}

(Received 22 December 2009 and accepted 25 January 2010, first published online

18 February 2010)

\begin{abstract}
The amplitude modulation of a finite amplitude drift wave by zonal flows in a non-uniform magnetoplasma is considered. The evolution of a nonlinearly coupled drift wave-zonal flow (DW-ZF) system is governed by a nonlinear equation for the slowly varying envelope of the drift waves, which drives (via the Reynolds stress of the drift wave envelope) the second equation for zonal flows. The nonlinear dispersion relation for the modulational instability of a drift wave pump is derived and analyzed. In a special case, the DW-ZF system of equations reduces to the cubic nonlinear Schrödinger equation, which admits localized solutions in the form of DW envelope solitons, accompanied by a shock-like ZF structure. Numerical solutions of the nonlinearly coupled DW-ZF systems reveal that an arbitrary spatial distribution of the DW rapidly decays into an array of localized drift wave structures, propagating with different speeds, that are robust and, in many respect, behave as solitons. The corresponding ZF evolves into the sequence of shocks that produces a strong shearing, i.e. multiple plasma flows in alternating directions.
\end{abstract}

It is widely recognized that large-scale sheared flows [1-3] (also referred to as convective cells (CCs) or zonal flows (ZFs)) play a very important role in regulating the cross-field turbulent transport in magnetically confined fusion plasmas. Typically, the $\mathrm{ZF}$ is a poloidally and toroidally symmetric structure with radial variation, whose potential fluctuation (in comparison with $T_{e} / e$, where $T_{e}$ is the electron temperature and $e$ is the magnitude of the electron charge) is much bigger than the relative zonal flow density perturbation (in comparison with the equilibrium plasma number density $\left.n_{0}\right)$.

In magnetically confined fusion plasmas, there exist free energy reservoirs in the form of density, temperature and magnetic field inhomogeneities, which are responsible for exciting the low-frequency (in comparison with the ion gyrofrequency), short-scale (of the order of the ion gyroradius or the ion sound gyroradius) DWlike fluctuations [4-6]. The linearly growing drift modes interact among themselves 
and attain large amplitudes in due course of time. The Reynolds stress of finite amplitude DWs, in turn, nonlinearly generates convective cells (CCs) and sheared flows/ZFs [7-13], via decay and modulational instabilities [8], respectively. Guo et al. [16] used the governing equations of [8] for the DW-CC turbulence system to investigate the radial spreading of the DW-ZF turbulence via soliton formation. There are recent review articles presenting the status of theoretical and simulation works [13], as well as experimental observations [14,15] concerning the dynamics of DW-ZF turbulence system.

In this paper, we study the amplitude modulation of finite amplitude drift wave packets by ZFs. We consider a non-uniform magnetoplasma in an external magnetic field $\hat{\mathbf{z}} B_{0}$, where $\hat{\mathbf{z}}$ is the unit vector along the $z$-axis in a Cartesian coordinate system and $B_{0}$ is the strength of the homogeneous magnetic field. The density gradient $\partial n_{0} / \partial x$ is along the $x$-axis. The dynamics of finite amplitude low-frequency (compared with the ion gyrofrequency $\omega_{c i}=e B_{0} / m_{i} c$, where $m_{i}$ is the ion mass and $c$ is the speed of light in vacuum) electrostatic DWs in the presence of ZFs is governed by [17]

$$
\begin{aligned}
\frac{\partial \phi}{\partial t}-V_{*} \frac{\partial \phi}{\partial y} & -\rho_{s}^{2}\left(\frac{\partial}{\partial t}+v_{i n}-0.3 v_{i i} \rho_{i}^{2} \nabla_{\perp}^{2}\right) \nabla_{\perp}^{2} \phi \\
& +\frac{c}{B_{0}}(\hat{z} \times \nabla \psi) \cdot \nabla\left(\phi-\rho_{S}^{2} \nabla_{\perp}^{2} \phi\right)=0,
\end{aligned}
$$

where $\phi$ is the DW potential, $V_{*}=C_{s} \rho_{s} / L_{n}, C_{s}=\left(k_{B} T_{e} / m_{i}\right)^{1 / 2}$ is the ion sound speed, $k_{B}$ is the Boltzmann constant, $T_{e}$ is the electron temperature, $\rho_{s}=C_{s} / \omega_{c i}$ is the ionsound gyroradius, $L_{n}=\left(\partial \operatorname{lnn}_{0} / \partial x\right)^{-1}$, $v_{i n}$ is the ion-neutral collision frequency, $v_{i i}$ is the ion-ion collision frequency and $\rho_{i}$ is the ion gyroradius.

The evolution of the ZF potential $\psi$ in the presence of the Reynolds stress of the DWs is governed by

$$
\left(\frac{\partial}{\partial t}+v_{i n}-0.3 v_{i i} \rho_{i}^{2} \nabla_{\perp}^{2}\right) \nabla_{\perp}^{2} \psi+\frac{c}{B_{0}}(1+\sigma)\left\langle(\hat{\mathbf{z}} \times \nabla \phi \cdot \nabla) \nabla_{\perp}^{2} \phi\right\rangle=0,
$$

where $\sigma=T_{i} / T_{e}$ in the ratio between the ion to electron temperatures. In the derivation of (1) and (2) we have neglected the small corrections due to the finite ion Larmor radius.

We now normalize the time and space variables by $\omega_{c i}^{-1}$ and $\rho_{s}$, as well as $\phi$ and $\psi$ by $k_{B} T_{e}$. In the normalized units, we can rewrite (1) and (2), respectively, as

$$
\left(1-\nabla_{\perp}^{2}\right) \frac{\partial \phi}{\partial t}-\frac{\rho_{s}}{L_{n}} \frac{\partial \phi}{\partial y}+\frac{\partial \psi}{\partial x} \frac{\partial}{\partial y}\left(\phi-\nabla_{\perp}^{2} \phi\right)=0,
$$

and

$$
\frac{\partial^{3} \psi}{\partial x^{2} \partial t}+\left\langle(\hat{\mathbf{z}} \times \nabla \phi \cdot \nabla) \nabla_{\perp}^{2} \phi\right\rangle=0,
$$

where, for convenience, we have neglected the contributions of ion-neutral and ion-ion collisions.

We now consider the amplitude modulation of the DW packet by ZFs. Accordingly, we introduce the two time and space scale analyses by supposing that the DW amplitude varies on the spatio-temporal scales $\left(\varepsilon x, \varepsilon^{2} t\right)$ of ZFs, where $\varepsilon \ll 1$ is a small 'bookkeeping parameter'. We use the envelope ansatz $\psi=\psi\left(\varepsilon x, \varepsilon^{2} t\right)$ and $\phi(x, y, t)=\varepsilon \varphi\left(\varepsilon x, \varepsilon^{2} t\right) \exp \left(-i \varepsilon \omega t+i k_{x} x+i k_{y} y\right)$, where $\varepsilon \omega$ is the normalized frequency of the linear drift wave, $\varepsilon \omega=k_{y} \rho_{s} / L_{n}\left(1+k_{\perp}^{2}\right), k_{\perp}^{2}=k_{x}^{2}+k_{y}^{2}$, and we assumed a 
weak inhomogeneity $\rho_{s} / L_{n}=\mathcal{O}(\varepsilon)$. Then, substituting in (3) $\partial / \partial t \rightarrow-i \varepsilon \omega+\varepsilon^{2} \partial / \partial t$, $\partial / \partial x \rightarrow i k_{x}+\varepsilon \partial / \partial x$ and $\partial / \partial y \rightarrow i k_{y}$, and with the accuracy to the leading order terms in the small parameter $\varepsilon,(3)$ and (4) take the following forms, respectively

$$
\left[\left(1+k_{\perp}^{2}\right)\left(\frac{\partial}{\partial t}-\frac{2 k_{x} \omega}{1+k_{\perp}^{2}} \frac{\partial}{\partial x}\right)+i \omega\left(1-\frac{4 k_{x}^{2}}{1+k_{\perp}^{2}}\right) \frac{\partial^{2}}{\partial x^{2}}\right] \varphi=-i k_{y}\left(1+k_{\perp}^{2}\right) \frac{\partial \psi}{\partial x} \varphi,(5)
$$

and

$$
\frac{\partial^{3} \psi}{\partial x^{2} \partial t}=2 k_{x} k_{y} \frac{\partial^{2}|\varphi|^{2}}{\partial x^{2}} \text {. }
$$

Using the notations $V_{g}=-2 k_{x} \omega /\left(1+k_{\perp}^{2}\right)=\partial \omega / \partial k_{x}$ and $P=\omega\left(1+k_{\perp}^{2}-4 k_{x}^{2}\right) /$ $\left(1+k_{\perp}^{2}\right)^{2}=-(1 / 2) \partial^{2} \omega / \partial k_{x}^{2}$, where $V_{g}$ and $P$ are identified as the $x$-components of the group velocity of the DW packet and the coefficient of the DW group dispersion, (5) and (6) take the simple form

$$
\left[i\left(\frac{\partial}{\partial t}+V_{g} \frac{\partial}{\partial x}\right)-P \frac{\partial^{2}}{\partial x^{2}}-k_{y} \frac{\partial \psi}{\partial x}\right] \varphi=0,
$$

and

$$
\frac{\partial \psi}{\partial t}=2 k_{x} k_{y}|\varphi|^{2}
$$

Equations (7) and (8) can be used to investigate the modulational instability of a constant amplitude drift wave pump and the long-term evolution of the amplitudemodulated DW packet and the excitation of the ZF structures by localized DW perturbations.

In the particular case when the zonal flow is a stationary function, traveling with a constant speed, (7) and (8) reduce to the standard nonlinear Schrödinger (NLS) equation, with a cubic nonlinearity, for the modulated DW envelope. In the reference frame moving with the group velocity, taking $\partial \psi / \partial t=-\left(U+V_{g}\right) \partial \psi / \partial x$ and $\left(\partial / \partial t+V_{g} \partial / \partial x\right) \varphi=\partial \varphi / \partial t^{\prime}$, where $U$ is the phase speed in the moving frame, we have

$$
\left(-i \frac{\partial}{\partial t^{\prime}}+P \frac{\partial^{2}}{\partial x^{2}}+Q|\varphi|^{2}\right) \varphi=0
$$

where the coefficient of the nonlinear term is a function of the velocity of propagation $U$, and it is given by $Q=-2 k_{x} k_{y}^{2} /\left(U+V_{g}\right)$. It is worth noting that the character of nonlinearity in (9) can be either focusing $(Q / P>0)$ or defocusing $(Q / P<0)$, depending on the signs and the magnitudes of the DW wave numbers and the phase velocity of the $\mathrm{ZF}$, viz.

$$
\frac{Q}{P}=\frac{2 L_{n} k_{x} k_{y}\left(1+k_{\perp}^{2}\right)^{3}}{\rho_{s}\left(U+V_{g}\right)\left(1+k_{\perp}^{2}-4 k_{x}^{2}\right)} .
$$

Equation (9) describes the parametric modulational instability of the drift waves, when the $\mathrm{ZF}$ is sought in the form $\psi=\exp [i \kappa(x-U t)]$ with a complex phase velocity $U$, as well as coherent nonlinear solutions in the form of solitary waves.

In the parametric regime, we seek the DW envelope as the sum of a constant DW pump and two DW sidebands, $\varphi=\varphi_{0}+\varphi_{+} \exp [-i(\Omega t-\kappa x)]+\varphi_{-} \exp [i(\Omega t-\kappa x)]+$ C.C., with $\left|\varphi_{+}\right| \sim\left|\varphi_{-}\right| \ll\left|\varphi_{0}\right|$. After the substitution into (9), we have

$$
\begin{gathered}
\left(-\Omega+P \kappa^{2}\right) \varphi_{+}+Q\left(\varphi_{0}^{*} \varphi_{+}+\varphi_{0} \varphi_{-}^{*}\right) \varphi_{0}=0, \\
\left(\Omega+P \kappa^{2}\right) \varphi_{-}^{*}+Q\left(\varphi_{0}^{*} \varphi_{+}+\varphi_{0} \varphi_{-}^{*}\right) \varphi_{0}^{*}=0 .
\end{gathered}
$$


Then, from the requirement that (11) and (12) have nontrivial solutions $\varphi_{-}, \varphi_{+} \neq 0$, we obtain the following dispersion relation

$$
\left(-\Omega+P \kappa^{2}+Q\left|\varphi_{0}\right|^{2}\right)\left(\Omega+P \kappa^{2}+Q\left|\varphi_{0}\right|^{2}\right)=Q^{2}\left|\varphi_{0}\right|^{4} .
$$

Making use of $\Omega / \kappa=U$, (13) simplifies to

$$
\left(P^{2} \kappa^{4}-\Omega^{2}\right)\left(\kappa V_{g}+\Omega\right)-4 P k_{x} k_{y}^{2} \kappa^{3}\left|\varphi_{0}\right|^{2}=0,
$$

which is a cubic algebraic equation for the frequency $\Omega$. It is readily solved as

$$
\Omega_{n}=\frac{\kappa}{3 A^{3}}\left[A^{6} e^{i \frac{2 \pi n}{3}}-V_{g} A^{3}+\left(V_{g}^{2}+3 P^{2} \kappa^{2}\right) e^{-i \frac{2 \pi n}{3}}\right],
$$

where $n=0,1,2$, and

$$
\begin{aligned}
A= & -54 P k_{x} k_{y}^{2}\left|\varphi_{0}\right|^{2}-V_{g}^{3}+9 P^{2} \kappa^{2} V_{g} \\
& +\left[\left(54 k_{x} k_{y}^{2} P\left|\varphi_{0}\right|^{2}-9 P^{2} V_{g} \kappa^{2}+V_{g}^{3}\right)^{2}-\left(V_{g}^{2}+3 P^{2} \kappa^{2}\right)^{3}\right]^{\frac{1}{2}} .
\end{aligned}
$$

Obviously, the frequency $\Omega$ may be complex. The threshold for the DW-ZF modulational instability, i.e. the DW amplitude $\left|\varphi_{0_{T}}\right|$ for which there is a double root of dispersion relation (14), is given by

$$
\left|\varphi_{0_{T}}\right|^{2}=\frac{ \pm\left(V_{g}^{2}+3 P^{2} \kappa^{2}\right)^{\frac{3}{2}}-V_{g}\left(V_{g}^{2}-9 P^{2} \kappa^{2}\right)}{54 P k_{x} k_{y}^{2}} .
$$

The solitary wave solution is easily constructed using the standard methods, e.g. by the ansatz $\varphi=\Phi(x-U t) \exp [i \Theta(x-W t)]$, where $\Phi$ and $\Theta$ are purely real functions of their arguments, and $U$ is the phase velocity of the $\mathrm{ZF}$ introduced earlier. In the simple case when $\Theta$ is a linear function of its argument, we can use a textbook expression for the NLS soliton, yielding

$$
\varphi=\sqrt{\frac{2 P}{Q}} K \operatorname{sech}[K(x-U t)] \times \exp \left\{-\frac{i U}{2 P}\left[x+\left(K^{2} P-\frac{U^{2}}{4}\right) t\right]\right\},
$$

and

$$
\psi=-\frac{4 P k_{x} k_{y}}{Q K U} \tanh [K(x-U t)] .
$$

Here $K$ is an arbitrary parameter, and the coupling coefficient $Q$ depends on the speed of the propagation of the wave envelope $U$, in contrast to the standard NLS soliton, for which it is a given constant. The solution presented in [16] is the special case of our (17) and (18), obtained for $U=0$.

It is worth noting that our basic equations (7) and (8), describing the coupled DWZF system, are not fully integrable and that the solitons, (17), are only particular solutions. In the general case, they might not be stable structures that emerge from arbitrary initial conditions. To check their stability, we studied the evolution of a localized DW packet in an initially quiescent plasma that did not feature any zonal flows. The system of (7) and (8) was solved numerically, assuming the absence of zonal flows at $t=0, \psi(x, t=0)=0$, and using the initial distribution of the drift waves in the form of a Gaussian, $\varphi(x, t=0)=\Phi \exp \left(-x^{2} / 2 L^{2}\right)$. Such initial conditions are well localized in space, but with a shape that is sufficiently different from the solitary structure (17) and (18), so that they can be regarded as 'near-arbitrary'. We used the 


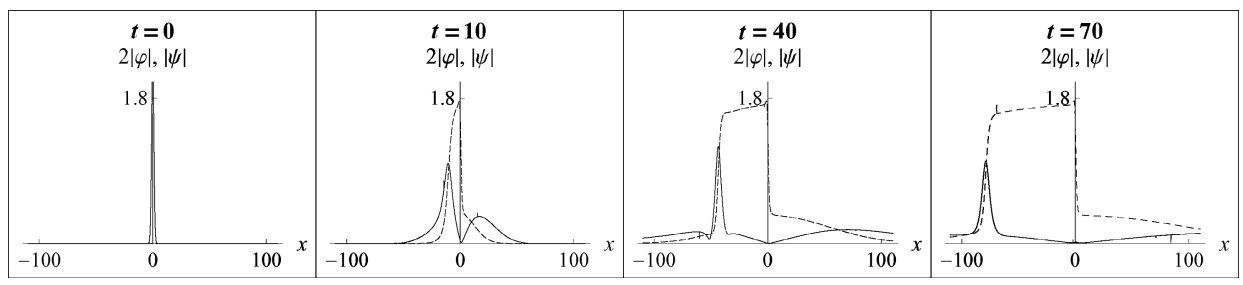

Figure 1. The evolution of a narrow drift wave packet (solid line) and the corresponding structure of the zonal flow (dashed line) in the case of a narrow initial DW distribution. The parameters in (7) and (8) are $V_{g}=-0.1, P=1, k_{y}=1, k_{x}=0.5$, the initial condition is given by $\psi(x, t=0)=0$ and $\varphi(x, t=0)=\Phi \exp \left(-x^{2} / 2 L^{2}\right)$, with $L=1, \Phi=1.2$ and the size of the computational box being $l=1000$.

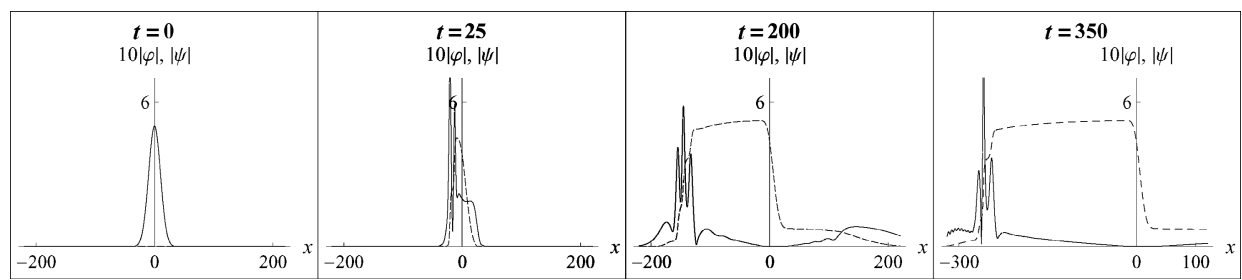

Figure 2. The evolution of the drift wave envelope (solid line) and the zonal flow (dashed line) in the case of an initial DW distribution with a medium width. All parameters are the same as in Fig. 1, except the initial DW distribution, whose parameters are $L=10$ and $\Phi=0.5$. The size of the computational box is $l=800$.

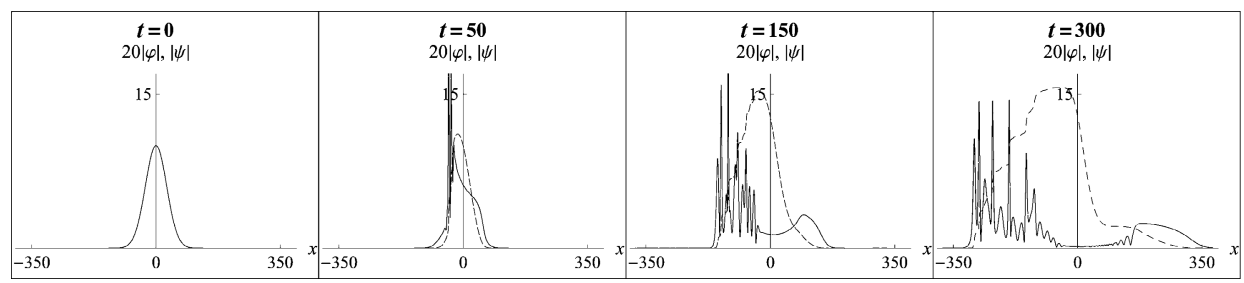

Figure 3. The evolution of the drift wave envelope (solid line) and the zonal flow (dashed line) in the case of a broad initial DW distribution. The parameters are the same as in Fig. 1 , except $L=30$ and $\Phi=0.5$. The size of the computational box is $l=1650$.

numerical method of lines, with a spatial discretization in the variable $x$, with $5-10$ thousand points. A periodic boundary condition was used with a sufficiently large computational box, and the calculations were terminated before any appreciable reflection from the boundary was detected. Three different initial distributions of the drift wave were studied, labeled as the narrow, the medium and the wide, and the results are displayed in Figs. 1-3.

For all initial distributions studied in this paper, the large amplitude packet of drift waves was split into two packets propagating in opposite directions. The one propagating opposite to the group velocity was subjected to the linear dispersion, and gradually spread to a width much broader than that of the initial packet. Conversely, the packet propagating in the direction of the group velocity did not 
undergo dispersion. For a narrow initial DW distribution, only one such peak was created that propagated for a long time with a constant speed and with very little change (see Fig. 1). For the medium and the wide initial packets, the portion of DW moving in the direction of the group velocity was almost immediately divided into several peaks, similar in shape to the sole peak in Fig. 1. The number of such peaks grew with the initial width of the packet. The peaks propagated with different speeds. In the case of the wide initial DW distribution, a number of overtaking collisions amongst them occurred, which they survived seemingly unchanged. In other words, these peaks demonstrated the features of true solitons, apart from the periodic oscillations of their amplitudes that were observed in some cases. The zonal flow was generated in a short time, $t \sim 1$. Initially, the ZF potential was also peaked, but it was later stretched by the moving DW wave packets. In the direction opposite to the group velocity, the slope of the ZF potential gradually decreased, together with the spreading of the corresponding DW packet due to dispersion. In the direction of the group velocity, the ZF potential evolved into a shock-like shape in the case of a single DW soliton, while in the presence of several DW solitons, it developed into a staircase-like sequence of shocks. Such ZF potential produces the usual pattern of strong shearing observed at the tokamak edge, i.e. the multiple poloidal plasma flows, having opposite directions.

In conclusion, we have studied the evolution of a nonlinearly coupled DW-ZF system that is governed by a nonlinear equation for the slowly varying envelope of the drift waves, which also drives, by the Reynolds stress, the second equation for zonal flows. The nonlinear dispersion relation for the modulational instability of a constant amplitude drift wave pump has been derived and solved for the growth rate and threshold. Furthermore, it has been shown that the full nonlinear system of equations reduces to a cubic NLS equation, which possesses localized solutions in the form of DW envelope solitons. Numerical studies have revealed that an arbitrary spatial distribution of the DW rapidly decays into the array of coherent structures, propagating with different speeds, that are robust and behave as true solitons, while the corresponding $\mathrm{ZF}$ evolved into the sequence of shocks, yielding multiple plasma flows in alternating directions.

\section{Acknowledgements}

This research was partially supported by the Deutsche Forschungsgemeinschaft through the project SH21/3-1 of the Research Unit 1048 and by the Serbian MNTR grant 141031. One of us (DJ) acknowledges the financial support from the DAAD.

\section{References}

[1] Lin, Z., Hahm, T. S., Lee, W. W., Tang, W. M. and White, R. B. 1998 Science 281, 1835.

[2] Hasegawa, A. 1985 Adv. Phys. 34, 1.

[3] Horton, W. and Hasegawa, A. 1994 Chaos 4, 227.

[4] Kadomtsev, B. B. 1965 Plasma Turbulence. New York: Academic.

[5] Horton, W. 1999 Rev. Mod. Phys. 71, 735.

[6] Weiland, J. 2000 Collective Modes in Inhomogeneous Plasma: Kinetic and Advanced Fluid Theory. Bristol, UK: IOP Publishing. 
[7] Sagdeev, R. Z., Shapiro, V. D. and Shevchenko, V. I. 1978 Zh. Eksp. Teor. Fiz. Pisma Red. 27, 361; [1978 JETP Lett. 27, 390; 1978 Fiz. Plazmy 4, 551; 1978 Sov. J. Plasma Phys. 4, 306].

[8] Shukla, P. K., Yu, M. Y., Rahman, H. U. and Spatschek, K. H. 1981 Phys. Rev. A 23, 321.

[9] Shukla, P. K., Yu, M. Y., Rahman, H. U. and Spatschek, K. H. 1984 Phys. Rep. 105, 227.

[10] Smolyakov, A. I., Diamond, P. H. and Malkov, M. 2000 Phys. Rev. Lett. 84, 491.

[11] Chen, L., Lin, Z. and White, R. 2000 Phys. Plasmas 7, 3129.

[12] Shukla, P. K. and Stenflo, L. 2002 Eur. Phys. J. D 20, 103.

[13] Diamond, P. H. et al. 2005 Plasma Phys. Control. Fusion 47, R35.

[14] Fujisawa, A. 2009 Nucl. Fusion 49, 013001.

[15] Tynan, G. R., Fujisawa, A. and McKee, G. 2009 Plasma Phys. Control. Fusion 51, 11301.

[16] Guo, Z., Chen, L. and Zonca, F. 2009 Phys. Rev. Lett. 103, 055002.

[17] Shukla, P. K. and Shaikh, D. 2009 Phys. Lett. A 374, 286. 